\title{
Die Mutter-Kind-Beziehung in der Mutterkuhhaltung
}

\author{
Summary \\ Title of the paper: Mother-infant-relationships in a beef suckler cow herd \\ A beef suckler cattle herd ( 25 cows, 23 calves) was observed on pasture for mother-infant behaviour. This \\ behaviour varied substantially in that 20 different suckling positions (with one, two or three calves suckling per \\ cow) were observed during 299 sucklings. \\ The frequent occuring cross suckling, the diversity of suckling positions as well as the behaviour \\ of cows during suckling are discussed in the context of the current literature. \\ In contrast to the literature, our results showed a higher incidence of cross suckling and a variety of suckling \\ positions, indicating a disturbed bonding between the cow and its calf during birth. \\ The preferred lying order of the herd showed parallel lying positions along the periphery of the pasture. \\ In conclusion, the results should encourage farmers to design their pastures according to the animal needs as \\ well as including regular herd observations into herd management schemes.
}

Key words: mother cow husbandry, mother-infant-relationships, nursing positions, lying order

\section{Zusammenfassung}

In einer Mutterkuhherde ( 25 Kühe, 23 Kälber) ergaben Verhaltensbeobachtungen eine hohe Variabilität des Mutter-Kind-Verhaltens. Die beobachteten 299 Saugakte verteilten sich auf insgesamt 20 verschiedene Säugestellungen, wobei ein, zwei bzw. drei Kälber je Kuh säugten. Das in der Herde auffallend häufige Auftreten von Fremdsäugen, die Vielfalt der Säugestellungen sowie das Verhalten der Kühe während des Säugens werden mit den Erkenntnissen der Literatur diskutiert. Diese Ergebnisse stehen im Widerspruch zu den in der Literatur gefundenen Angaben und sind außerdem ein Anzeichen für Störungen innerhalb der Bindung Kuh-Kalb. Die Ursachen sind in den Störungen bereits während des Geburtsablaufes zu suchen.

Die Liegeordnungen der Herde zeigten eine Bevorzugung von parallelen Strukturen entlang von Begrenzungen der Weideflächen und unterliegen zeitlichen Veränderungen.

Die Ergebnisse sollen den Tierhalter anregen, über eine Strukturierung der Weideflächen die Haltung tiergerechter zu gestalten sowie die regelmäßige Beobachtung der Herde mit in das Haltungsmanagement einzubeziehen.

Schlüsselwërter: Mutterkuhhaltung, Mutter-Kind-Beziehung, Säugestellungen, Liegeordnung

\section{Einleitung}

Trotz der Einbrüche auf dem Rindfleischmarkt ist aufgrund des in Deutschland zu verzeichnenden Trends einer verstärkten Extensivierung weiterhin mit einer steigenden Anzahl von Mutterkühen zu rechnen. So stieg die Anzahl der Mutterkühe in den vergangenen vier Jahren von 396.000 (1992) auf 593.000 (Viehzählung 12/1996). Die 
Zahl der mutterkuhhaltenden Betriebe erhöhte sich von 39.000 (1992) auf 50.700 (1996) - Angaben nach BML 1997 -. Trotz dieses Trends und obwohl die Mutterkuhhaltung eine seit Jahrzehnten angewandte Haltungsform für Extensivstandorte ist, existieren wenige Untersuchungen, die sich mit der Vielfalt des Verhaltens bei dieser Haltungsform beschäftigen. So werden die häufig allgemein getroffenen Aussagen zu den ethologischen Erfordernissen des Weidemanagements einer modernen Mutterkuhhaltung, deren Ziel ökologisch produziertes Qualitätsfleisch ist, nicht mehr gerecht. BAUMGARTNER (1996) sieht die Überwachung der Mutterkuhherde während des Abkalbens nicht nur aus wirtschaftlichen Interessen, sondern auch im Sinne des Tierschutzes als unbedingt nötig an. Um die geforderte tierschutz- und verhaltensgerechte Haltung realisieren zu können, ist es nötig, den Kenntnisstand auf diesem Gebiet zu erweitern. Hierzu müssen in den Herden zuerst die Bedingungen für eine stabile Mutter-Kind-Beziehung geschaffen werden. Gerade hierin liegt aber das Problem: Die ohnehin gegenüber der Stallhaltung geringe Betreuungsintensität führt zu einem geringen Mensch-Tier-Kontakt. Das erklärt, daß sich seitens der Betreuungsperson(-en) Wissenslücken zu den konkreten sozialen Bindungen innerhalb der Herde ausbilden.

Die vorliegende Untersuchung soll - anhand der gewonnenen systematischen Verhaltensbeobachtungen in einer Versuchsherde - ein Beispiel für die Vielfalt des MutterKind-Verhaltens darstellen.

Ähnlich wie bei Wildrindern, sondern sich auch in Mutterkuhherden die Kühe kurz vor der Geburt ab. Sie gebären ihr Kalb versteckt im Gebüsch oder im hohem Gras und legen ihr Kalb dort ab (SCHLOETH, 1961). Dies dient dem Schutz (vor allem vor ranghohen Kühen) und damit der ungestörten Prägung der Kuh auf ihr Kalb (REINHARDT, 1980). Das Belecken des Kalbes nach der Geburt dient nicht nur der Entfernung der Eihäute und des Fruchtwassers sowie der Anregung zur Durchblutung (NAAKTGEBOREN, 1972), sondern auch der geruchlichen Prägung der Kuh auf das Kalb (DERENBACH, 1981). Nach der Geburt bleibt die Kuh drei bis vier Tage beim Kalb, kehrt dann aber zur Herde zurück und trifft es nur noch zum Säugen. Die Kälber vereinigen sich zu Kälberherden (Kindergärten), bei denen sich immer einige Kühe der Herde zum Schutz aufhalten (REINHARDT, 1980).

\subsection{Die „Bindung“ innerhalb der Mutter-Kind-Beziehung}

ROTH (1978), der sich mit der Mutter-Kind-Beziehung (MKB) beim Rind intensiv beschäftigte, nannte für das Zustandekommen dieser Bindung zwei Eigenschaften:

1. Die Bereitschaft des Muttertieres, zu einem Jungtier derart in Kontakt zu treten, daß daraus eine MKB entstehen kann. Diese Bereitschaft wird gefördert durch:

- die Erfahrung im Gebären und im mütterlichem Verhalten sowie

- den Zustand des Jungtieres und seinem Verhalten. 
2. Die Selektivität des Muttertieres, diese bildet sich sehr schnell, wahrscheinlich noch während des Geburtsvorganges heraus. Dabei verhält sich das Muttertier normalerweise nur den Jungtieren gegenüber pflegend, sorgend, säugend und schützend, zu denen die MKB besteht.

So stuft VOIGT (1996) die Ammenkuhhaltung bereits als nur bedingt verhaltensgerecht ein, da oft nur eine Duldung des Kalbes durch die Amme und keine echte Bindung erreicht wird. Außerdem stellen beim Fremdsäugen sowohl die Auseinandersetzungen des Kalbes mit den anderen Kälbern als auch die Abwehrbewegungen der Amme einen ständigen Streßfaktor dar.

\section{2.}

Tiere und Methodik

\section{Tiere und Weidefuhrung}

In eigenen Untersuchungen wurde das Verhalten einer Mutterkuhherde, bestehend aus 25 Kühen (dreijährig, SMR bzw. SMR x Fleckvieh) mit 23 Kälbern, videodokumentiert. Die Aufnahmen erfolgten an 21 Tagen, verteilt über die Monate Juli - September, in der Zeit von 09.00 bis $12.00 \mathrm{Uhr}$. In dieser Zeit befand sich die Herde ganztägig auf der Weide. Alle 2 - 3 Tage wurden die Tiere vom Weidewart auf ein neues Weidestück umgetrieben. Die Größe der Weide wurde dabei willkürlich ausgewählt. Das genutzte Weideareal war von Waldstreifen und Straßen umsäumt.

Die Klimawerte des Areals zeigt Tabelle 1.

Tabelle 1

Mittelwerte, Minima und Maxima von Temperatur, Luftdruck und Windgeschwindigkeit (21 Beobachtungstage) (Means, minimum and maximum of temperature, air pressure and wind speed (21 days))

\begin{tabular}{r|r|r|c}
\hline Klimafaktor & $\overline{\mathrm{x}}$ & Min & Max \\
\hline Luftdruck (hPa) & 998,0 & $992,3 \quad(4 . \mathrm{BT})$ & 1007,1 (5. BT) \\
\hline relative Luftfeuchte (\%) & 55,0 & 31,6 (5. BT) & 81,0 (12. BT) \\
\hline Temperatur $\left({ }^{\circ} \mathrm{C}\right)$ & 22,2 & $14,1(18 . \mathrm{BT})$ & $30,8(8 . \mathrm{BT})$ \\
\hline Windgeschwindigkeit $(\mathrm{m} / \mathrm{s})$ & 8,5 & $0,6(1 . \mathrm{BT})$ & 12,3 (17. BT) \\
\hline
\end{tabular}

\section{Verhaltensbeobachtungen}

Um das Verhalten der untersuchten Herde nicht $\mathrm{zu}$ beeinflussen, wurde diese von Forsthochständen mit einer Videokamera gefilmt. Dabei erfolgte in 5min-Intervallen eine Gesamtaufnahme der Herde und in der Zwischenzeit wurde das Verhalten von Einzeltieren als Nahaufnahme gefilmt.

Das Videomaterial wurde nach folgenden Gesichtspunkten ausgewertet:

- Erfassung aller Saugakte und der Säugestellung unterschieden in:

- Säugen $1 \mathrm{Kalb} / \mathrm{Kuh}$

- Säugen 2 Kälber/Kuh

- Säugen 3 Kälber/Kuh

- Verhalten der Kuh während des Säugens (Analkontrolle, bewegungslos Stehen, Gra- 
sen, Harnen, Lecken eines fremden Kalbes, Säugen an anderer Kuh)

\section{Liegeordnungen}

In 5min-Intervallen erfolgte die Anfertigung einer Umrißzeichnung über die Liegeordnung und -struktur der Herde vom Standbild des Videorekorders. Dafür wurden die ausgewählten Weideflächen anhand ihrer Umzäunungen vermessen. Die Zaunpfähle dienten dabei am Videostandbild als Maßstab für die Entfernungen der Tiere zueinander bzw. für die Ausrichtung und Entfernung zum Elektrozaun. Ermittelt wurden die Entfernungen der Tiere zueinander und die Blickrichtung. Die Figuren der Liegeordnungen entstanden durch das Verbinden der kürzesten Entfernung zwischen zwei Tieren auf der Zeichnung.

Die statistische Auswertung erfolgte nach Übernahme der Daten mittels des Statistikprogrammes SAS. Zur Testung der Einflußfaktoren „Zeit“ , „Kuh“ bzw. „Kuh/Zeit“ wurde die mehrfaktorielle Varianzanalyse (Typ III) ausgewählt.

3. Ergebnisse und Diskussion

\section{Säugen}

Als Grundlage für die Auswertung dienten die während des Versuchszeitraumes in der Herde beobachteten 299 Saugakte, die sich auf das Säugen von 1 - 3 Kälbern/Kuh verteilten (Tab. 2).

Tabelle 2

Verteilung der Saugakte und Säugestellungen auf die Anzahl der säugenden Kälber/Kuh (Distribution of sucklings and suckling positions for the number of calfes/cow)

\begin{tabular}{lccc}
\hline Kälber/Kuh & $\begin{array}{c}\text { Saugakte } \\
\text { (n) }\end{array}$ & $\begin{array}{c}\text { Häufigkeit } \\
(\%)\end{array}$ & $\begin{array}{c}\text { Säugestellungen } \\
\text { (n) }\end{array}$ \\
\hline eins & 256 & 85,6 & 5 \\
zwei & 38 & 12,7 & 10 \\
drei & 5 & 1,7 & 5 \\
\hline
\end{tabular}

Aufgrund der Vielzahl der beobachteten Säugestellungen, sollen nur die am häufigsten aufgetretenen genannt und diskutiert werden.

Für das Säugen ein Kalb/Kuh wurden insgesamt fünf verschiedene Säugestellungen festgestellt. Die verkehrtparallele Stellung war dabei in Übereinstimmung mit SAMBRAUS (1971) am häufigsten (56,5 \%) zu beobachten (Tab. 3). Die meisten Kühe blieben bei dieser Stellung während des Säugens bewegungslos Stehen (65,2 \%) und nur $11,1 \%$ der Kühe führten die anale Kontrolle durch (Abb. 1). MAGNAC et al. (1991) stellten an vier Bisonkühen nur bis 28,0\% Stehen+Lecken+Riechen gegenüber den Kälbern an der Gesamtaktivität während des Säugens fest.

In der Stellung von hinten durch die Hinterbeine der Kuh (mit 23,8 \% zweithäufigste Stellung) wurde am häufigsten an bewegungslos stehenden Kühen $(31,7 \%)$ gesäugt. Somit ist diese Stellung nicht nur als eine Alternative des Säugens beim Weiterlaufen der Kuh zu werten (SCHLOETH, 1958). Die Vielfalt des Verhaltens der Kälber beim Milchstehlen dokumentiert auch das nicht nur ausnahmsweise (DERENBACH, 1981) 
Tabelle 3 Verteilung der Säugestellungen für das Säugen ein Kalb/Kuh (Distribution of suckling positions for suckling one
calf/cow)

\begin{tabular}{lcc}
\hline Säugestellung & Saugakte & Häufigkeit \\
& (n) & $(\%)$ \\
\hline verkehrtparallel & 144 & 56,3 \\
von hinten durch die Hinterbeine & 61 & 23,8 \\
von hinten um eines der Hinterbeine & 7 & 2,7 \\
seitlich von hinten & 3 & 1,2 \\
im rechten Winkel & 41 & 16,0 \\
\hline
\end{tabular}

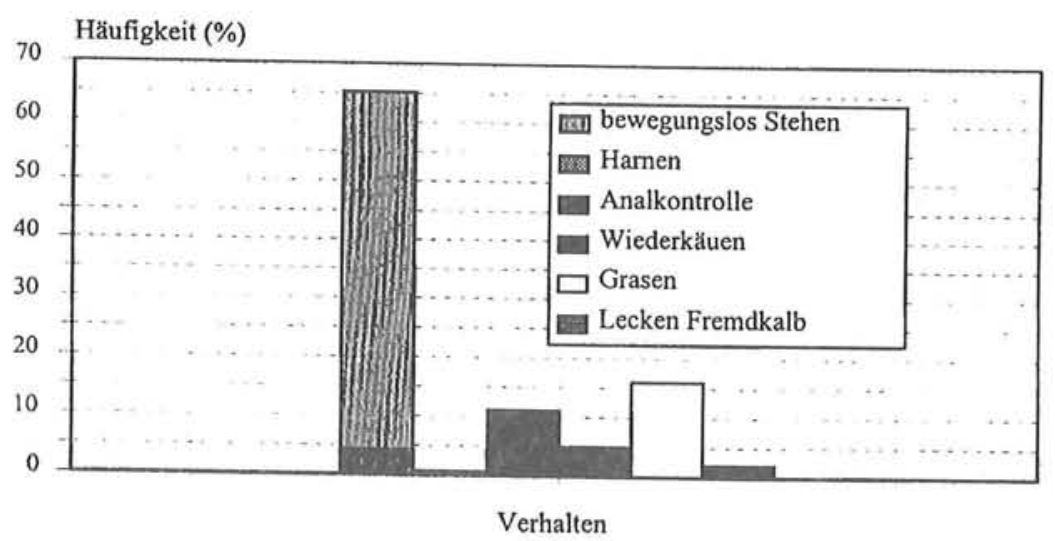
Abb. 1: Verhalten der Kühe während des Säugens in verkehrtparalleler Stellung (Behaviour of cows during
suckling in antiparallel position)

Auftreten der Stellungen von hinten um eines der Hinterbeine der Kuh und seitlich von hinten. RICE (1995) dokumentierte die gleichen Stellungen bei Nilgiri-Tahren. Anhand des Verhaltens von Böcken, die ebenfalls diese Stellungen zeigten, entwickelte er die These, daß sich aufgrund dieser ähnlichen Verhaltensweisen Teile des Sexualverhaltens aus dem Mutter-Kind-Verhalten entwickelten. Schlußfolgernd vermutet er, daß diese Parallelen auch bei anderen taxonomischen Einheiten zu finden sind.

Das bereits von SCHLOETH (1958) und DERENBACH (1981) beobachtete Säugen in der Stellung im rechten Winkel zur Kuh trat in der Versuchsherde mit $16 \%$ auf.

In der Herde konnte auch Säugen von zwei Kälbern/Kuh in zehn verschiedenen Stellungen beobachtet werden. Am erfolgreichsten waren dabei die Stellungen, bei denen neben einem verkehrtparallel säugendem Kalb, sich jeweils ein weiteres verkehrtparallel (13,1 \%) bzw. durch die Hinterbeine der Kuh (36,8 \%) säugendes Kalb hinzugesellte. Nach KILEY-WORTHINGTON und DE LA PLAIN (1983) ist die Stellung mit zwei verkehrtparallel säugenden Kälbern/Kuh als nicht erfolgreich beim Milchstehlen anzusehen, da ein Erkennen des fremden Kalbes über die Geruchskontrolle durch das Muttertier möglich ist. ARAVE und ALBRIGHT (1981) halten diese Stellung für geeignet dafür, daß die Kuh ein „Pflegekalb“ akzeptiert. Das Säugen zweier Kälber nebeneinander im rechten Winkel zur Kuh (2,6\%) bzw. das zweite Kalb säugt im rechten Winkel um eines der Hinterbeine der Kuh $(5,3 \%)$ herum, ist wie von 
REINHARDT und REINHARDT (1981) beobachtet als erfolgreiche Variante des Milchstehlens zu bewerten. Das zweite Kalb steht hierbei im Geruchsschatten des ersten Kalbes und kann damit nicht durch die Kuh als Fremdkalb identifiziert werden.

Für die fünf beobachteten Säugestellungen beim Saugen drei Kälber/Kuh konnten der Literatur keine Angaben entnommen werden. Säugestellungen für vier Kälber/Kuh, wie von RITTER (1961) sowie BIETZ et al. (1974) beschrieben, kommen den bei drei Kälbern/Kuh beobachteten jedoch sehr nahe.

Das gehäufte Auftreten von bis zu drei säugenden Kälbern/Kuh zeigte einen wesentlich größeren Anteil an fremdsäugenden Kälbern als erwartet an. Somit bedarf die von GRAUVOGL (1992) gestellte Forderung, daß Kühe, die das Fremdsäugen von Kälbern dulden aus der Herde entfernt werden, einer kritischen Überprüfung. Bei der veränderten Lage in der Rindermast, kann unter Umständen jedes natürlich aufgezogene Kalb einen finanziellen Gewinn für den Halter darstellen. Andererseits bedeutet Fremdsäugen natürlich einen Energieverlust für die betroffene Kuh. Diese Ressourcen fehlen dem eigenen Kalb. Untersuchungen von GOLZE et al. (1989) an 623 Ammenkühen und 842 Kälbern zeigten, daß die eigenen Kälber der Ammen bei einem Besatz von zwei Kälbern/Amme, $319 \mathrm{~g}$ weniger tägliche Zunahmen zu verzeichnen hatten. Dabei reduzierte sich ebenfalls sowohl die Lebendmasse der Kuh als auch des Fremdkalbes.

\section{Liegeordnung der Herde}

Anhand der Umrißzeichnungen von Liegeordnungen des Kindergartens der Kälber und einiger innerhalb der Herde konnten Rückschlüsse über deren bevorzugte Struktur und Ausrichtung während des Liegens gewonnen werden (Abb. 2).

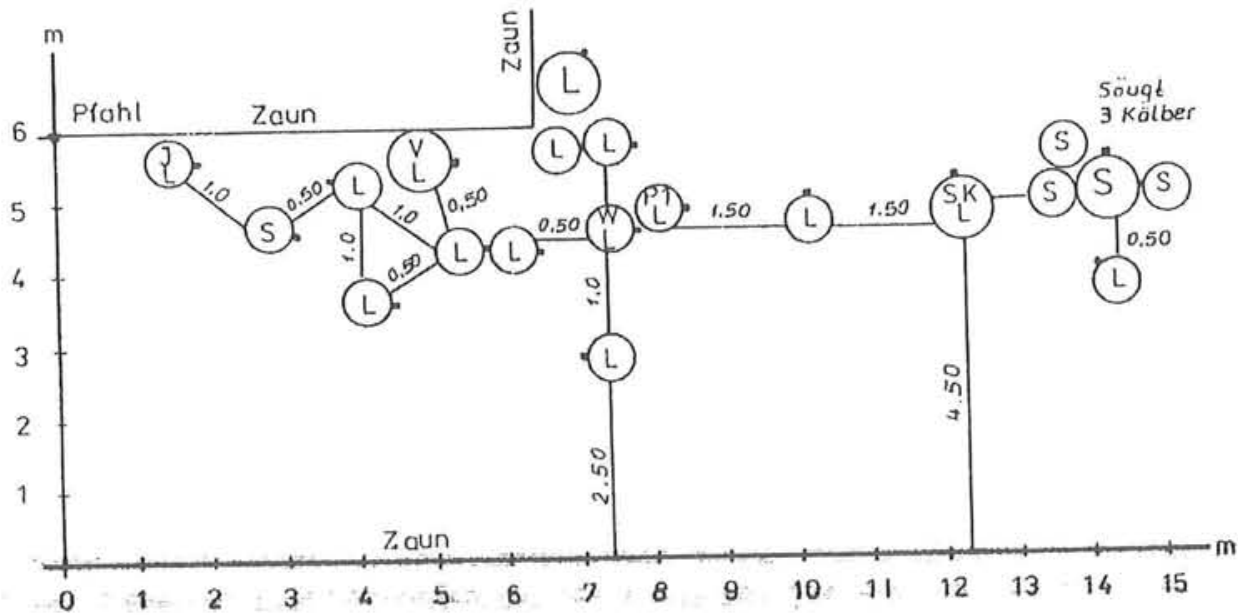

Abb. 2: Darstellung einer typischen Liegeordung (Typical lying order) großer Kreis $=$ Kuh, kleiner Kreis $=$ Kalb, Kreisspitze $=$ Blickrichtung (big circle $=$ cow, little circle $=$ calf, circle point $=$ line of vision) $\mathrm{S}=$ Tiere stehen auf der Stelle, $\mathrm{L}=$ liegende Tiere, weitere Buchstaben = individuell beobachtete Tiere $(\mathrm{S}=$ standing animals, $\mathrm{L}=$ lying animals, other letters = individual observed animals)

Die Umrisse zeigten, daß in der Versuchsherde eindeutig lineare Strukturen des ge- 
meinsamen Liegens bevorzugt wurden. Dabei zweigen von einer linearen Hauptstruktur, die von nebeneinanderliegenden Tieren gebildet wird, astartige Nebenstrukturen ab (Abb. 3). Bei sinkender Tierzahl der zusammenliegenden Tiere werden zuerst die Nebenstrukturen der Liegeordnungen abgebaut.

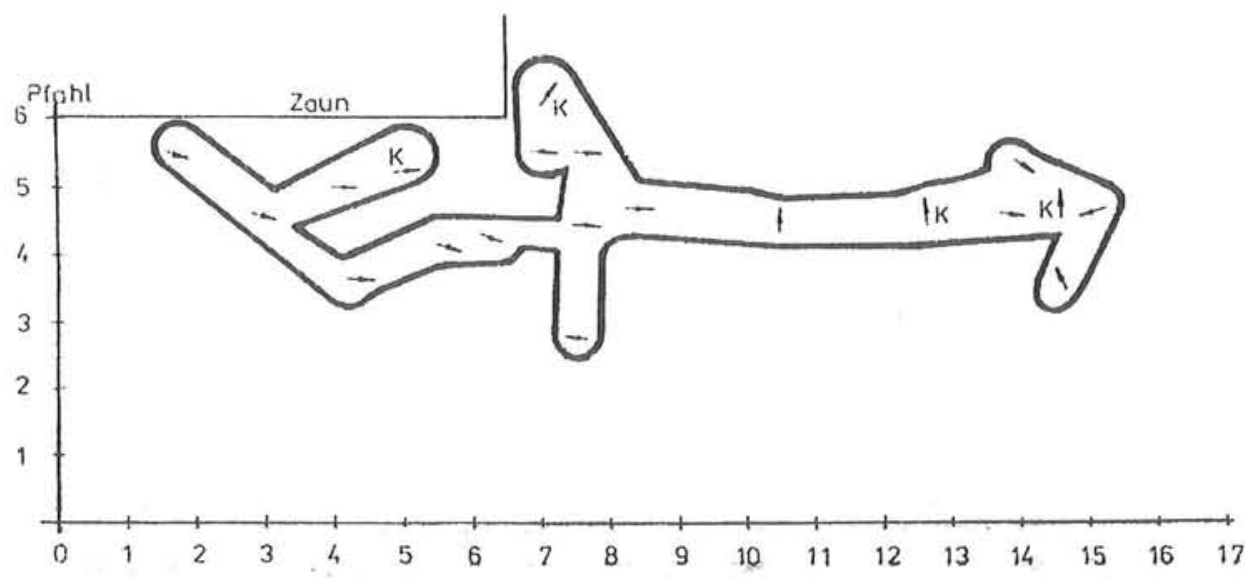

Abb. 3: Umrißzeichnung der Liegeordung, die sich aus den Kreisdarstellungen ergeben (An outline of lying order. A result of the circles)

Diese Nebenstrukturen unterliegen zeitlichen Veränderungen, die durch das Dazukommen mit nachfolgendem Abliegen bzw. dem Aufstehen und anschließenden Weglaufen einzelner Tiere entstehen. Die Konstanz der Liegeordnungen hält bis zu $30 \mathrm{~min}$ an. Der Einflußfaktor „Zeit“ konnte zumindest für die Liegeordnungen eines Beobachtungstages statistisch gesichert werden (mehrfaktorielle Varianzanalyse, $\alpha=0,05$ ). Für die Faktoren „Kuh“ bzw. „Kuh / Zeit“ konnten keine gesicherten Ergebnisse erzielt werden.

Die festgestellten Liegestrukturen zeigten Tendenzen zur parallelen Ausrichtung gegenüber angrenzenden Straßen und Waldstreifen. Ähnliche, auf Linearität beruhende Strukturen beschrieben BACKHAUS (1959) bei Lelwel-Kuhantilopen (A. buselaphus lelwel) und WALTHER (1964) bei Weißschwanzgnus (C. gnou).

Die parellele Ausrichtung der Herde während des gemeinsamen Liegens gegenüber angrenzenden Geländestrukturen, zeigt eine gewisse Übereinstimmung mit der parallelen Ausrichtung von Kleinen Kudus (T. imberbis) innerhalb des Geheges zu den Gehegegegenständen (WALTHER, 1964).

\section{Schlußfolgerungen}

Die festgestellte Vielfalt der Säugestellungen innerhalb der untersuchten Mutterkuhherde läßt im Vergleich mit der Literatur den Eindruck entstehen, daß die Varietät des Säugens noch nicht in seiner gesamten Häufigkeit des Auftretens erfaßt wurde.

Damit die Geburt und die nachfolgende Prägung zwischen Muttertier und Kalb unge- 
stört verlaufen können, ergeben sich für den Tierhalter folgende Konsequenzen:

Die den Tieren aufgezwungenen Umweltbedingungen - in Form von großenteils baumund strauchlosen Weiden - sind durch die Einbeziehung von Struktur z. B. von Vegetation so zu gestalten, daß Rückzugsmöglichkeiten für die kalbende Kuh und Abliegeplätze für die neugeborenen Kälber entstehen. Die Notwendigkeit dieser Strukturen zeigten die umfangreichen Untersuchungen von LIDFORS (1994) sowie die von RAASCH und KÖNIG (1998) beschriebenen Gefahren durch lying out der Kälber auBerhalb der Weide. Selbst bei Gruppenhaltung der Mutterkühe im Stall mit hoher Besatzdichte drehen diese ihr Becken zur Wand, um ungestört zu sein, sobald die Geburt bevorsteht (GRÜNBERG, 1996).

Da der weitere Aufzuchterfolg großenteils von der Mütterlichkeit der einzelnen Kühe abhängt, sollte die regelmäßige Tierbeobachtung und die daraus resultierende Kenntnis über die Bindungen innerhalb der Herde ein fester Bestandteil des Managements innerhalb der Mutterkuhhaltung sein.

\section{Literatur}

ARAVE, C. W.; ALBRIGHT, J.L.:

BACKHAUS, D.:

Cattle behaviour. J. Dairy Sci., Champaign, Ill. 64 (1981), 1318 - 1329

Beobachtungen an Antilopen und Beobachtungen uber das Freileben von Lelwel-Kuhantilopen (Alcelaphus buselaphus HEUGLIN, 1877) und Gelegenheitsbeobachtungen an Sennar-Pferdeantilopen (Hippotragus equinus bakeri HEUGLIN, 1863). Z. Săugetierkunde 24 (1959), 1-34

BAUMGARTNER, G.:

Extensive Tierhaltung und Tierschutz. 3. Trenthorster Kolloquium, 5. / 6.12.1996, „Über die Haltung von Rindern mit Saugkảlbern (Mutterkuhhaltung) als extensive Tierhaltungsform, Landbauforschung Völkenrode, Sonderheft 177, $231-239,1997$

BIETZ, G.; NEUMANN, W.; ZUPP, W.

Haltung und Verhalten von vorgenutzten Färsen und saugenden Kälbern. Tierzucht, Berlin 28 (1974), $531-534$

BML : Mehr Mutterkuhhalter in Deutschland. Tierärztl. Umschau 12 / 97, 742, 1997

DERENBACH, J.:

Untersuchungen zum Saugverhalten neugeborener Kălber in der Mutterkuhhaltung. Univ. Stuttgart-

Hohenheim, Diss., 1981

GOLZE, M.; SCHMALFUB, R.; SCHWARK, H.J.:

Ammenkuhhaltung - Eine Möglichkeit der Absicherung der Mastrinderbestände zur Erhöhung der Schlachtrinderproduktion. 20. Jahrestag. Sektion TV der KMU Leipzig, „Züchterische Maßnahmen zur Verbesserung des genetischen Potentials und zur Realisierung hoher Tierleistungen", Leipzig, 14. I 15.02.1989, $358-363$

GRAUVOGL, A.: Mutterkühe tierartgerecht halten. Tierzilchter (1992), 35 - 37

GRÜNBERG, W.:

Untersuchung zur Eignung der Rinderrasse Deutsch Schwarzbunt (DSB) für eine ganzjăhrige Außenhaltung in besonderer Berücksichtigung der Abkalbung. Tierärztliche Hochschule Hannover, Diss. med. vet., 1996

KILEY-WORTHINGTON, M. ; DE LA PLAIN, S.:

The behaviour of beef suckler cattle (Bos taurus). Tierhaltung Bd. 14, Birkhäuser Verlag Basel-BostonStuttgart, 1983

LIDFORS, L.:

Mother - young behaviour in cattle. Parturition, development of cow-calf attachment, suckling and effects of separation. Rapport 33, Skara, Schweden, 1994 
MAGNAC, M.P.; ALTMANN, D.; GONZALEZ, G.:

Maternal behaviour and independence of the calves within a captive group of American Bison. In: Proc. Int. Congr. „Ongules / Ungulates“, Toulouse 1991

NAAKTGEBOREN, C.:

Die Umstellung auf das eigenständige Leben, Teil II: Das Geburtsverhalten. Image 48, 1972, 16

RAASCH, M.-L.; KÖNIG, I.: Lying out - eine Gefahr für Kälber auf der Weide? In: DVG/ TVT-Fachtagung „Tierschutz und Nutz-

REINHARDT, V.: Untersuchung zum Sozialverhalten des Rindes. Tierhaltung Bd. 10, Birkhäuser Verlag Basel-BostonStuttgart, 1980

REINHARDT, V.; REINHARDT, A. :

Ethologische und reproduktionsphysiologische Aspekte der Mutterkuhhaltung ohne ZwangsentwöhRICE, C. G. nung der Kälber. In: Bericht 3. Arb.tag. AGHST, BVA Gumpenstein, 2./3.10.1981, 31 - 38

RITTER, H.C.:

On the origin of sexual displays in caprids. Z. Säugetierkunde 60 (1995), 53 - 62

ROTH, E.:

Ammenkuhhaltung im Laufstall. Tierzüchter 13 (1961), 148 - 51

Vorläufige Mitteilung über Geburtsverlauf und Kälberzusatz beim Hausrind (Mutter- und Ammenkuhhaltung). In: Aktuelle Arbeiten zur artgemäßen Tierhaltung 1978. KTBL-Schrift 240, 169-91

SCHLOETH, R.:

Über die Mutter-Kind-Beziehungen beim halbwilden Camargue-Rind. Säugetierkundl. Mitt. 6 (1958) $145-50$

SCHLOETH, R.:

Das Sozialleben des Camargue-Rindes. Z. Tierpsychol. 18 (1961), 574 - 627

SAMBRAUS, H. H.:

VOIGT, K.:

Zum Mutter-Kind-Verhalten der Wiederkäuer. Berl. Münch. Tierärztl. Wschr. 84 (1971), 24 - 27

Artgemäße Kälberaufzucht. Ökologie \& Landbau 24 (1996), 53 - 55

WALTHER; F.:

Zum Liegeverhalten des Weißschwanzgnus (Connochaetes gnou ZIMMERMANN, 1780). Z. Säugetierkunde 31 (1966), 1-16

Eingegangen: 30.07 .1998

Akzeptiert: 11.02.1999

Anschriften der Verfasser

Dr. DIRK SCHÄFFER

Institut für Tierzucht und Tierhaltung mit Tierklinik, Professur für Tierhygiene und Tierklinik

Landwirtschaftliche Fakultät der Martin-Luther-Universität Halle-Wittenberg

Emil-Abderhalden-Str. 27/28

D-06108 Halle (Saale)

E-Mail: schaeffer@landw.uni-halle.de

Prof. Dr. EBERHARD v. BORELL

Institut für Tierzucht und Tierbaltung mit Tierklinik

Landwirtschaftliche Fakultät, Martin-Luther-Universität Halle-Wittenberg

A.-Kuckhoff-Str. 35

D-06108 Halle (Saale)

Dr. RALF-BERND LAUBE

Institut für Lebensmittelhygiene

Veterinärmedizinische Fakultät

Universität Leipzig

Sitz: Albrecht-Daniel-Thaer-Institut für Nutztierwissenschaften Leipzig, e. V.

Fichtestr. 28

D-04275 Leipzig 


\section{Forschungsinstitut für die Biologie landwirtschaftlicher Nutztiere}

Im Forschungsinstitut für die Biologie landwirtschaftlicher Nutztiere (FBN) in Dummerstorf (Mecklenburg-Vorpommern) ist zum nächstmöglichen Zeitpunkt der Dienstposten der/des

\section{Leiterim/Leiters}

zu besetzen.

Die Einstellung erfolgt bei Erfüllung der laufbahnrechtlichen Voraussetzungen nach

\section{BesGr. B 3 BBesO.}

Das FBN ist eine Stiftung des öffentlichen Rechts, Mitglied der Wissenschaftsgemeinschaft Gottfried Wilhelm Leibniz, mit rd. 240 Mitarbeiterinnen/Mitarbeitern.

Aufgabe des Instituts ist die Grundlagenforschung auf dem Gebiet der Biologie landwirtschaftlicher Nutztiere. Die Bereiche der Forschung in diesem Institut umfassen Populationsbiologie und Züchtungsforschung, Molekularbiologie, Fortpflanzungsbiologie, Physiologische Grundlagen der Tierhaltung, Muskelbiologie und Wachstum, Ernährungsphysiologie und Biometrie.

Anforderungen:

- international fachlich herausragend ausgewiesene/r Wissenschaftlerin/Wissenschaftler mit abgeschlossener Habilitation oder vergleichbarer Qualifikation in einem der genannten Arbeitsgebiete

- Erfahrungen in der Forschung am landwirtschaftlichen Nutztier

- soziale Kompetenz

- Fähigkeit, mit Innovation, hohem persönlichen Einsatz und Organisationstalent eine größere interdisziplinär arbeitende Forschungseinrichtung zu leiten

Schwerbehinderte werden bei gleicher Eignung bevorzugt berücksichtigt, von ihnen wird nur ein Mindestmaß an körperlicher Eignung verlangt. Bewerbungen von Frauen sind besonders erwünscht.

Bewerbungen mit tabellarischem Lebenslauf, Lichtbild, Zeugnisabschriften, Übersicht über die bisherige Tätigkeit sowie Liste der Veröffentlichungen sind bis zum 31. Mai 1999 zu richten an:

Den Vorsitzenden des Kuratoriums des Forschungsinstituts für die Biologie landwirtschaftlicher Nutztiere 